\title{
Entrelacs
}

ENTRELACS Cinéma et audiovisuel

$10 \mid 2013$

Le Toucher

\section{Ce qu'imaginer veut dire}

\section{Gérard Leblanc}

\section{(2) OpenEdition \\ Journals}

Édition électronique

URL : http://journals.openedition.org/entrelacs/489

DOI : $10.4000 /$ entrelacs.489

ISSN : 2261-5482

Éditeur

Éditions Téraèdre

\section{Référence électronique}

Gérard Leblanc, «Ce qu'imaginer veut dire », Entrelacs [En ligne], 10 | 2013, mis en ligne le 11

septembre 2013, consulté le 19 avril 2019. URL : http://journals.openedition.org/entrelacs/489; DOI : 10.4000/entrelacs.489

Ce document a été généré automatiquement le 19 avril 2019

Tous droits réservés 


\title{
Ce qu'imaginer veut dire
}

\author{
Gérard Leblanc
}

1 Ce texte répond à une commande du musée Alexandre Dumas. Il concerne les relations que l'écriture dumasienne entretient avec le cinéma (et inversement). Il m'a semblé toutefois pouvoir prendre place dans ce numéro dans la mesure où il questionne le rôle de l'imaginaire dans son rapport au sensible, et particulièrement au toucher, à travers la littérature, le cinéma et les dispositifs de réalité virtuelle.

$2 \mathrm{Au}$ cours d'une "Causerie avec mes lecteurs» (Le Mousquetaire, 10 décembre 1853), Alexandre Dumas évoque l'imaginaire d'un de ses collaborateurs de théâtre et amis, Gérard de Nerval : «De temps en temps, lorsqu'un travail quelconque l'a fort préoccupé, l'imagination, cette folle du logis, en chasse momentanément la raison, qui n'en est que la maîtresse ; alors la première reste seule, toute puissante, dans ce cerveau nourri de rêves et d'hallucinations, ni plus ni moins qu'un fumeur d'opium du Caire, ou qu'un mangeur de hatchis d'Alger, et alors, la vagabonde qu'elle est, le jette dans les théories impossibles, dans les livres infaisables... » (fin d'extrait de citation). Aussi profonde soit la sympathie ou même l'admiration qu'il éprouve pour Nerval et sa poésie, Dumas ne se laissera jamais enfermer dans la forme d'imaginaire qu'il attribue à Nerval. Quels que soient les tours et détours pris par son imagination romanesque et théâtrale, il saura raison garder. Son imaginaire aura les limites de la raison historique ou de la raison tout court. Il agencera ses livres de telle façon que son lecteur puisse s'y retrouver tout en échappant à luimême. Le récit aventureux l'emporte sur l'aventure de l'écriture.

3 Dumas n'en gardera pas moins, tout au long de sa vie, un goût prononcé pour le fantastique intérieur, comme en témoigne par exemple son roman La femme au collier de velours (1850). Devenu le personnage principal de son roman, l'écrivain romantique allemand Hoffmann s'exclame : «Je suis fou ! Et Dumas de commenter : «L'exclamation d'Hoffmann n'avait rien d'exagéré : cette faible cloison qui, chez le poète exerçant outre mesure ses facultés cérébrales, cette faible cloison, disons-nous, qui, séparant l'imagination de la folie, semble parfois prête à se rompre, craquait dans sa tête avec le bruit d'une muraille qui se lézarde. » Mais Hoffmann n'est pas le double de Dumas comme peut l'être la folie dans l'expérience de dédoublement chez Nerval. Dumas reste maitre de 
son écriture et n'entend pas se laisser entraîner là où il ne veut pas aller. Néanmoins, il se fait fort d'y conduire le lecteur.

Dumas et Nerval se retrouvent pourtant sur un point majeur : ils entendent faire passer l'imaginaire dans la vie. Les deux écrivains et amis, à la fois proches et lointains ont, chacun à sa façon, fait de leur vie une aventure. Une aventure qu'ils ont porté l'un et l'autre aux limites du possible.

5 Il est entendu que la littérature a influencé le cinéma mais quelle littérature ? Question qui se double immédiatement d'une autre, complémentaire : la littérature a influencé le cinéma mais quel cinéma? Alexandre Dumas a influencé un cinéma qui tendait à rendre l'écriture transparente à la représentation. Celle qui s'appuyait sur les mots pour mieux les traverser. Celle qui permettait au lecteur de visualiser ce qui était écrit sans s'arrêter sur les mots. Dans l'écriture poétique, les images ne peuvent pas se séparer des mots. Il s'agit de mots-images ou d'images-mots. L'écriture pré-cinématographique à la Dumas, une écriture scénaristique avant la lettre du cinéma, génère des images dans le cerveau $\mathrm{du}$ lecteur et on les dirait aussi précises et vivantes que si elles eussent été cinématographiées. Il s'agit d'images concrètes, presque matérielles. Lèverait-on la main qu'on aurait l'impression de pouvoir les toucher du doigt.

6 Donner à lire pour imaginer, c'est montrer par l'écriture, c'est faire voir ce qui serait même interdit à la vue. «Eh bien! C'est dans une chambre située au premier étage d'une maison dont les fenêtres donnent de biais sur le portail de l'église des Jésuites, que nous allons conduire nos lecteurs... ». Au- delà de l'acte de lecture, le lecteur est invité à regarder ce que la lecture lui suggère. La scène mise en spectacle s'éloigne de l'écriture, elle se présente à nous, elle nous aspire dans une chaîne d'actions et de réactions qui nous laisse bouche bée. Sommes-nous encore en train de lire ? Peut-être avons-nous suspendu la lecture, nous nous trouvons là où l'auteur nous a entrainés, nous sommes immergés dans la scène, nous y participons.

7 Croire en la réalité de ce qu'on écrit. Se représenter la scène mieux, en devenir acteur, vivre l'action que l'on décrit. Il va de soi qu'un écrivain ne peut décrire sans écrire mais l'écriture tend vers la transparence de la description. La description ne vaut pas par ellemême, elle ne vaut pas non plus par son intérêt littéraire bien qu'elle puisse en avoir un, elle vaut d'abord par sa capacité à susciter des images dans le cerveau du lecteur. Il faut pouvoir se représenter une scène et se la représenter de telle façon que l'on puisse en devenir un des acteurs, serait-ce sous la forme d'une présence muette, celle de l'observateur. Le lecteur voit ce qu'il lit mieux, il le vit. Il ne se contente plus de tourner les pages comme un lecteur qui sait qu'il est en train de lire. La lecture est débordée par des représentations qui se concrétisent dans des images. Alexandre Dumas ne raconte pas des histoires à dormir debout mais à s'agiter assis. Il ne s'agit plus seulement de mots imprimés sur la page. Les mots ouvrent sur la scène où le lecteur est invité de façon pressante à prendre place et où, une fois qu'il y a pris place, à ne plus tenir en place. Cette forme de représentation requiert la participation c'est-à-dire la présence. La gestuelle du lecteur, par ses mouvements incontrôlés, témoigne du fait qu'il vit à l'intérieur de la représentation, qu'il s'y est engouffré pieds et poings liés. Il est pris par la scène, il ne s'appartient plus. La représentation s'est muée en présence active.

8 Bien entendu, l'action reste dans le champ de l'imaginaire. On imagine qu'on agit. Le roman interdit l'action et le cinéma ne la permet pas davantage. Mais une certaine littérature comme un certain cinéma aiguisent le désir d'action du lecteur ou du spectateur tout en sachant pertinemment qu'ils ne peuvent le satisfaire. Ils jouent même 
de cette impossibilité pour favoriser l'identification aux personnages de la fiction. Je m'identifie à un personnage parce qu'il agit à ma place et selon un mode d'action que j'aurais choisi si j'avais pu agir moi-même. Il est mon représentant, mieux, il me représente tel que j'aurais pu agir à sa place si j'avais eu la possibilité de le faire. J'adhère à son comportement, à ses paroles, à ses actes. Il est devenu un autre moi-même.

Écriture héritée du théâtre, sans aucun doute, avec de multiples rebondissements d'intrigue. L'écrivain raconte et met en scène ce qu'il raconte. La description n'est rien de moins qu'un dispositif de mise en scène. En ce sens, Dumas préfigure le cinéma, du moins un certain type de cinéma fondé sur le narratif et l'aventure. Il opère, par la médiation du roman - historique ou non - le passage du théâtre au cinéma. La scène dumasienne est trop à l'étroit sur la scène théâtrale en dépit des décors mobiles qu'il a conçus. Car les actions s'enchaînent les unes aux autres sur de multiples scènes en perpétuel mouvement. Elles n'ont d'autre lieu que le mouvement lui-même, un mouvement qui ne connaît pas l'arrêt, un mouvement continu, celui du cinéma.

Est-on au spectacle ou dans le spectacle? C'est la ligne de démarcation que Dumas nous fait franchir en favorisant le déploiement de l'imaginaire du lecteur dans l'action. Cette action ne peut être circonscrite dans un espace scénique matérialisé qui viendrait lui fixer un cadre. Aussi surprenant et stupéfiant soit-il, le coup de théâtre n'équivaut pas à un coup de cinéma bien que le cinéma puisse parfaitement l'intégrer (le coup de cinéma relève essentiellement du truquage). Le cinéma n'est pas arrimé à un espace scénique même si l'industrie cinématographique, très vite, l'a encadré sur une surface d'écran qui aurait pu être beaucoup plus vaste. Les propositions ne manquaient pas à cet égard, à commencer par l'écran circulaire de Grimoin Sanson. Dès la fin du XIXe siècle, le spectateur aurait pu être entouré par un cercle d'images, continues ou discontinues. C'était en tout cas techniquement possible.

11 L'excitation verbale fait lever des images qui se situent au-delà de la représentation : des images à vivre. Sont-elles de chair et de sang quand le cerveau du lecteur, au comble de l'excitation, se révèle incapable de tracer une ligne frontière entre ce qu'il lit et ce qu'il vit. Les images sont faites de la chair et du sang des personnages auxquels le lecteur s'identifie faute de pouvoir agir lui-même. L'observateur qu'il est d'abord ne reste jamais longtemps en situation d'extériorité. Il se mêle aux personnages, il prend parti pour tel ou tel contre tel ou tel autre. L'observateur se transforme très vite en acteur.

Écrire dans l'excitation du désir d'écrire. On en vit sans doute (on feuilletonne) mais davantage encore on vit ce qu'on écrit. L'écriture fait partie de la vie, elle permet à la vie d'avoir lieu dans une certaine aisance et splendeur, mais elle est loin d'en constituer le seul sel et aliment. L'écriture est un festin mais Dumas se repait tout autant de toutes les chairs possibles et imaginables. Son écriture est celle d'un grand bavard qui ne s'arrête guère de parler que pour d'autres plaisirs au moins aussi intenses, avec une attention toute particulière, malgré les références et les soumissions convenues à la religion, pour les nourritures qui réjouissent et ravissent le corps.

13 L'enthousiasme, la gourmandise d'écrire. On n'est jamais rassasié. Il en faut toujours plus et plus pimenté encore. Des nourritures roboratives qui à la fois tiennent au corps et font décoller l'imaginaire. L'écriture fait à tel point partie de la vie que le discours à la première personne s'entremêle continûment au récit. Le moindre déplacement est prétexte à écrire qu'on se déplace et que les récits qu'on en tire valaient bien le déplacement. Dumas est l'auteur de ses livres, il en est également l'acteur et pas seulement en tant qu'écrivain. Écrire et voyager, dans le temps comme dans l'espace, ne 
font qu'un. Où que l'on soit d'ailleurs, écrire échauffe le cerveau à moins qu'il ne soit déjà échauffé par la bonne chère et les bons vins. Écrire fait partie de la vie d'une autre manière encore. Il s'agit bien de dégager des revenus, de feuilletonner en somme, et l'importance des dialogues chez Dumas a partie liée avec le mode de rémunération des feuilletonistes de son époque, payés à la ligne et non au nombre de signes. On tire plus facilement à la ligne avec les phrases courtes d'un dialogue vif et enlevé. Mais la contrainte économique ne réduit jamais Dumas au rôle de plumitif ou de tâcheron. La brièveté des dialogues est certes rémunératrice mais elle contribue aussi à affuter les joutes verbales et à les rendre finalement plus tranchantes conformément à l'esprit du duel.

14 Gagner en comptant pour dépenser et se dépenser sans compter. L'argent n'a pas le temps de s'accumuler, il est déjà dépensé. Écrire vite et beaucoup est la condition sans laquelle on ne saurait dépenser sans compter. Les personnages sont calqués sur ce modèle économique. Ils agissent vite et beaucoup. Profusion de situations et d'actions. L'action d'écrire ne s'arrête jamais ou plutôt se renouvelle jour après jour. Il n'y a pas de fin programmée du feuilleton ou du moins pas de fin programmée à l'activité de feuilletoniste.

Une écriture en perpétuel mouvement. Jamais de repos. L'écriture ne fait jamais relâche.

Il faut que la main coure sur le papier comme un animal débusqué par un chasseur. Manger les phrases, dévorer les paragraphes. Arpenter la page comme un chasseur de phrases. L'appétit d'écrire fait les récits appétissants.

L'écriture est un festin qui s'incarne dans la chair de personnages qui se mettent à exister devant et avec nous. Et de projeter ces personnages dans des situations aussi imprenables que peut l'être une vue profonde et sans limites. Le récit entremêlera des intrigues à rebondissements et suspens multiples. Il s'agira bientôt pour le lecteur d'essayer de prendre l'écriture de vitesse. On aura de plus en plus hâte de se transporter au-delà du paragraphe que l'on est en train de lire. À peine a-t-on connaissance de ce qui se passe ou vient de se passer que l'on se demande, avec une excitation toujours plus fébrile, ce qui va se passer. On ne revient jamais en arrière. On est porté en avant au point d'anticiper ce qui va arriver et qui nous ménage encore bien des surprises. Le lecteur ne sait pas toujours à quel écrivain il a affaire.

On projette l'action devant soi, on s'efforce de l'anticiper, on la déroule comme une scène à transformations multiples. La précipitation est la règle. On projette l'action devant soi et on s'y projette tout entier. La page se traverse comme un paysage saturé de nuages et de chemins piégés. Les éclairs n'illuminent jamais qu'une partie de la scène. L'orage garde hauts tenus ses secrets. Et le lecteur de naviguer à vue dans les brouillards et les brouillons de l'Histoire.

18 Échauffer le lecteur, exciter sa curiosité et surtout son imagination. Provoquer un imaginaire de la curiosité. De l'extraordinaire, oui, mais de l'extraordinaire validé par l'histoire. Entre mille exemples, d'Artagnan a bel et bien existé. Bien entendu, il n'existe pas chez Dumas de la même façon que Rossellini, par exemple, le fait exister dans La prise du pouvoir par Louis XIV (1966). Rossellini en fait un exécuteur des hautes œuvres du jeune Louis XIV (arrestation de Fouché) mais un exécuteur qui reste malgré tout un exécutant, obéissant et plutôt effacé. Rien d'héroïque dans l'action représentée, le simple accomplissement, net et sans bavures, d'un rôle et d'une fonction. Or, Dumas insiste sur l'aspect héroïque de ses personnages. Ils jouent le rôle qui leur est dévolu par l'Histoire mais l'intensité de leur action excède ce rôle. L'Histoire est moins un enjeu qu'un terrain 
de jeu où l'on éprouve la vie dans le risque de la perdre. C'est ainsi que l'on s'éprouve vivant. velours : «Peut-être le lecteur se demandera-t-il, ou plutôt nous demandera-t-il »), celuici ne se permettrait pas de déranger celui-là si, à la suite du Diable boiteux de Lesage, il ne se proposait pas de lui ouvrir le toit des maisons les mieux protégées, s'il n'ambitionnait pas de le mettre au fait des secrets de l'Histoire en épousant les tours et détours de la vie de ses acteurs et de ses actrices. Le lecteur de Dumas est un privilégié. Il est un privilégié parce que Dumas lui-même dispose d'un privilège qu'il n'hésite pas à partager avec son lecteur : « Grâce au privilège que nous possédons de compte à demi avec le Diable boiteux, et qui nous donne la faculté de pénétrer dans chaque maison fermée... » (Joseph Basalmo). «[...] Nous arrogeant le privilège du Diable boiteux, nous venons de lever le plafond de sa chambre et de le montrer à nos lecteurs debout, appuyé à la muraille, immobile derrière son rideau, haletant, les yeux fixés sur le portail de l'église des Jésuites. » (Il s'agit du personnage de l'écrivain Hoffmann dans La femme au collier de velours.) La pulsion scopique du lecteur ne cesse d'être sollicitée et satisfaite. Il peut tout voir : voir ce qui échappe à la vue de l'homme ordinaire, voir même ce dont il ne soupçonnait pas l'existence. Vous allez voir ce que vous allez voir, tel est le programme perpétuellement relancé et accompli de Dumas. L'écriture ne serait rien si elle ne se prolongeait pas dans des vues dont beaucoup s'apparentent à des visions. Il arrive certes que ces visions, et plus fréquemment qu'on ne dit, soient la résultante de métaphores littéraires mais le plus souvent l'écriture constitue une sorte de rampe de lancement pour l'imaginaire du lecteur. À lui maintenant de passer de l'autre côté, dans la scène.

Cà y est. On est devenu celui qui agit. On agit soi-même. Est-ce une réalité ou une simple impression ? À moins que ce ne soit une subtile combinaison des deux. Tantôt je lis, tantôt je m'oublie comme lecteur. Pourtant, je continue à lire. Et puis non, sans m'en apercevoir, j'ai interrompu ma lecture. Mes yeux ne parcourent plus la page d'un regard haletant. C'est un autre regard qui se substitue à celui du lecteur, le regard de quelqu'un qui est entré en action.

Vivre ce qu'on lit en agissant à l'intérieur de la représentation, en s'identifiant aux personnages ou bien encore à leurs actions. Il existe une dynamique de l'écriture qui fait qu'on se représente dynamiquement une scène, une situation, des personnages. On se projette dans une action au point de la vivre. Dumas dispose de cette très rare capacité de faire naître la vie de l'écriture. 

cinématographique à l'aune de la charpente narrative de ses romans, certainement. Il faudrait des heures et des heures de film pour en épouser la complexe architecture sans pouvoir prétendre d'ailleurs l'épuiser. Le format cinéma, tel qu'il s'est figé et rigidifié au cours de son histoire ( 90 minutes pour un film de durée standard) ne correspond pas au temps élastique d'un livre qu'on prend, qu'on déprend et qu'on reprend. À moins que le cinéma ne reprenne à la littérature la formule du feuilleton. On peut feuilletonner au cinéma aussi bien qu'en littérature : c'est le serial. À l'orée de la guerre de 14-18, Louis Feuillade l'a prouvé avec sa remarquable adaptation en cinq épisodes du Fantômas de Pierre Souvestre et de Marcel Allain (cinq épisodes seulement parce que le sixième allait avoir lieu dans les tranchées). Dix épisodes des Vampires suivront en 1915, soit 7 heures et 20 minutes de film. L'adaptation des Trois mousquetaires en 12 épisodes de 60 minutes réalisée par Henri Diamant Berger en 1921, certes moins inventive, participe elle aussi de cette formule. Malheureusement, le réalisateur ne put obtenir le concours de Douglas Fairbanks pour tenir le rôle de d'Artagnan, celui-ci ne jurant le cinéma que par Hollywood. Au cours de la même année 1921, Fairbanks tournait d'ailleurs sous la direction de Fred Niblo une version américaine des Trois mousquetaires. feuilleton. Peut-être parce que le feuilleton connaissait un relatif déclin dans l'édition littéraire mais surtout parce que la séance de cinéma assignait une place et une durée standards au film de fiction. Relayant le cinéma, la télévision a tenté de reprendre la formule sans atteindre de grandes réussites si l'on excepte L'homme sans visage de Georges Franju (1973-74, 8 fois 52 minutes).

Adapter Dumas au cinéma ? La question s'est posée très vite, dès que le cinéma s'est mis à raconter des histoires à visée spectaculaire. L'écriture de Dumas ne s'apparentait-elle pas déjà au cinéma au point qu'il n'est pas incongru de se demander si, une poignée d'années plus tard, Dumas lui-même ne serait pas devenu cinéaste? Ses livres ne fourmillent-ils pas d'aventures toutes plus palpitantes les unes que les autres? Ne revendiquent-ils pas le mouvement comme principe de vie et d'écriture? Le mouvement culmine dans la forme " cape et d'épée », la plus fréquemment reprise au cinéma. La figure du duel, qui se décompose en multiples positions et postures, en constitue l'action la plus emblématique.

Les adaptations de Dumas au cinéma sont légion. C'est à mon sens Abel Gance qui en est le plus proche par son désir de faire participer le spectateur à 1 'action (de l'incorporer à l'action, écrit-il), de le transformer en spectaCteur. Car c'est bien en ce point que le bât de l'écriture blesse Dumas. Il donne à voir des personnages qui agissent mais il s'agit encore et toujours d'une représentation. La littérature donne à imaginer mais le cinéma, jusqu'à un certain point, concrétise cet imaginaire. Il dispose de l'image et, qui mieux est, de l'image en mouvement. Il lui donne forme, consistance et présence. Le cinéma ne serait-il pas le point d'aboutissement de l'écriture dumasienne?

29 Comme on le sait, la poursuite a constitué la première figure de l'aventure au cinéma et, très vite, il s'est agi de l'étoffer en l'enchâssant dans des récits à péripéties de plus en plus sinueuses et compliquées. Les livres de Dumas, ses romans historiques en premier lieu, 
constituaient un fond patrimonial de premier choix et servi tout chaud pour élaborer un des premiers genres cinématographiques: le film d'aventures. Action, mouvement, gestuelle enfiévrée, vitesse, déplacements permanents, énergie, dynamisme, rapidité, rythme, cadence. Et par-dessus tout, l'enthousiasme de vivre en agissant. Le cinéma pouvait se retrouver dans Dumas, s'y retrouver jusque dans son horreur de l'arrêt du mouvement qui, pour Dumas, s'apparentait à la mort. Le cinéma pouvait se retrouver aussi dans la conception dumasienne du dialogue. N'a-t-il pas inventé la forme la plus achevé du dialogue : le champ/contre-champ? Les joutes verbales pouvaient s'y déployer tout à leur aise et la figure du duel s'y épanouir tout autant.

L'écriture de Dumas est rapide mais encore bien trop lente à ses yeux : « Tout ce que nous venons de raconter en quinze ou vingt lignes s'était passé aussi rapidement que l'éclair ». Que serait une écriture uniquement composée d'éclairs? Il est clair en tout cas qu'on ne pourrait plus tirer à la ligne. C'est un cinéma déchiré d'éclairs et de précipitations en tout genre qu'anticipe l'écriture dumasienne.

Il ne faudrait pas croire cependant que le cinéma, parce qu'il montre des images en mouvement, ne donne plus à imaginer. Concrétisant l'imaginaire littéraire, le cinéma pourrait être tenté de ne plus solliciter l'imagination. Ce serait évidemment renoncer à lui-même. L'imagination ne se laisse pas résorber par la perception. Quand il en va ainsi, le spectateur s'ennuie, il arrive même qu'il s'endorme. Il préfère rêver. En avoir plein les yeux n'est pas suffisant : il faut aussi en avoir plein l'imaginaire. Le cinéma est un moyen de porter l'imaginaire plus avant ou il existe à peine.

Dans les dispositifs interactifs, on confond souvent l'action physique (le fameux clic sur le clavier de l'ordinateur ou les manettes des consoles de jeux) avec l'imaginaire de l'action (car «l'usager », comme il est convenu de le nommer, interagit avec des actions qui relèvent malgré tout de l'ordre de l'imaginaire puisqu'il ne les accomplit pas lui-même). Or, la perception se situe entre sens de la vue et sens haptique. Elle se rapporte aux sensations qu'elle filtre, atténue et éteint le plus souvent. C'est dans cet entre-deux que l'imaginaire pointe de façon irréductible. La vue fait naître le désir de toucher: non le passage à l'acte mais le désir de l'acte. On se rapproche par la vue du toucher sans jamais l'accomplir. La limite de l'art se situe bien dans un au-delà du regard. Le regard veut percevoir au-delà de ce que l'art lui propose. Il veut finir par saisir l'objet de son émotion. D'où la place incontournable de l'imaginaire pour figurer le sens haptique, cet au-delà du regard.

L'image filmique qui ne cultive pas l'imaginaire ne fait que consigner des représentations préexistantes, souvent plus pauvres que l'imaginaire du lecteur travaillé par la lecture, ce qui est le cas pour beaucoup d'adaptations cinématographiques des livres de Dumas. Je n'ai pas l'intention d'établir ici un palmarès, de choisir telle adaptation des Trois mousquetaires contre telle autre. Bien entendu, on peut être plus sensible aux adaptations de Max Linder (dans le registre de la parodie burlesque) ou de Richard Lester (à cause de sa légèreté dansante) qu'à celles de André Hunebelle ou de Bernard Borderie. Certaines sont plus fidèles à la lettre du texte, d'autres à son esprit. Mais la plupart de ces adaptations achoppent sur un point essentiel. Elles ne parviennent pas à utiliser les moyens du cinéma pour enrichir l'imaginaire littéraire dumasien. Elles restent dans le registre de l'illustration.

S'agirait-il alors de transposer Dumas dans des univers qui seraient spécifiques à chaque cinéaste qui s'emparerait de son œuvre, comme le fit par exemple Patrice Chéreau pour son adaptation de La reine Margot (1994) ? Il est vrai que les auteurs de films qui ont puisé 
dans son œuvre sont jusqu'à nos jours assez rares. On ne se préoccuperait plus alors de la fidélité ou de l'infidélité de l'adaptation puisqu'il s'agirait d'une transposition du point de vue d'un autre auteur et que le film en dirait autant, sinon davantage, de l'univers du cinéaste que de celui de Dumas.

Ni adaptation ni transposition. Pour exister au cinéma, il me semble que Dumas n'a besoin ni d'illustrateurs ni d'auteurs de films. Il a besoin d'auteurs de cinéma qui remettent en question les limites du cinéma comme il a éprouvé lui-même les limites de la littérature. C'est en ce point que nous rencontrons le Cyrano et d'Artagnan d'Abel Gance (1964). Bien que Gance se soit abondamment inspiré de Dumas, en particulier pour la composition du personnage de d'Artagnan et des mousquetaires, il ne s'agit en aucun cas d'une adaptation d'un de ses livres. Mais on peut y retrouver, transportées au cinéma, les préoccupations de Dumas en littérature.

L'objectif de la caméra est l'œil mécanique par la médiation duquel on regarde et par la médiation duquel on est regardé. L'objectif est au centre de toute médiation, il est ce par quoi le regard circule, de la salle à l'écran et de l'écran à la salle. D'où l'importance que Gance attribue à la sensation de sa présence pour le spectaCteur. En témoigne l'écriture scénaristique du cinéaste dans la séquence de "Un contre cent ». Le grand duc Noicaron, à la tête d'une armée nocturne de hiboux, de chouettes et de chauve-souris, est supposé venir de l'objectif. La coquille de l'épée de Cyrano est presque dans l'objectif avant qu'elle ne sorte de l'objectif. Cyrano se fendrait-il à fond sur un adversaire? Sans doute, mais il se fend d'abord à fond sur l'objectif. Mieux: son épée part comme un éclair de l'objectif et touche la tempe de la Colombe. Auparavant, la Colombe avait jeté ses gants vers l'objectif.

Un personnage peut d'ailleurs représenter un « objectif » pour d'autres personnages, au sens où il est "visé " par eux. C'est ainsi qu'une foule s'apprête à déferler sur l'objectif Cyrano. Peu de temps après, Cyrano lance une bombe en direction de l'objectif. En une autre occasion, Cyrano ouvre les bras en direction de la caméra pour recueillir les hommages émerveillés de ses amis après son incroyable combat victorieux.

Et par-dessus tout, des fulgurances, des éclairs. Nous voyons tout cela, écrit Gance, en un éclair : des plans se succèdent et s'entremêlent, des plans de 3 secondes, puis de 2 secondes, puis de 1 seconde. Et Gance d'écrire dans une formule synthétique : « La pointe de l'épée fulgurante de Cyrano sortant de l'objectif touche en éclair la tempe du chef des Ecorcheurs. "

Ce n'est pas que l'objectif de la caméra enregistre «objectivement » ce qui se présente devant lui. C'est même tout le contraire. L'objectif est un tenant lieu du spectaCteur, il fusionne avec lui. Il est à l'origine de l'action ou il en est le résultat. C'est de l'objectif que les mobiles proviennent, c'est vers l'objectif qu'ils se dirigent. Il est ce par quoi la subjectivité du spectaCteur est saisie.Tout part de lui et tout revient vers lui. Le spectaCteur se trouve bien au centre de l'action ou, plus exactement, il est comme traversé par elle. Il se situe sur une ligne frontière perpétuellement mouvante et instable, celle qui sépare un champ de son contre-champ. Il est dans la collure. Mais pour que l'objectif joue pleinement son rôle, ne faudrait-il pas crever l'écran d'un définitif coup d'épée ? Le rôle dévolu à l'objectif vise à supprimer l'écran: le projet d'Abel Gance consiste bien à abolir toute forme de médiation entre l'action représentée et le spectaCteur.

Dans la perspective sonore selon Gance, les sons viennent tantôt de l'écran et tantôt de la salle, par le truchement de haut-parleurs disséminés dans la salle, idéalement derrière 
chaque fauteuil. C'est encore un moyen d'abolir la séparation entre l'écran et la salle et de faire se répondre les deux espaces, de les conjuguer en somme. On peut considérer à cet égard qu'un fauteuil équipé d'un haut-parleur est l'équivalent sonore d'un objectif de caméra selon Gance. Les évènements sonores viennent d'un côté comme de l'autre ce qui contribue à les intégrer dans la réalité vécue par le spectaCteur. Qu'ils viennent de la salle ou de l'écran, les évènements sonores se situent sur le même plan de réalité. Il faudrait être de bien mauvaise foi pour nier la réalité selon laquelle le spectaCteur est bien assis dans une salle de cinéma. Arrimée à chaque fauteuil, la source sonore qui en provient contribue à faire accéder chaque motif narratif au même niveau de réalité. On remarquera enfin que l'écoute est à la fois collective et singulière, aucun spectacteur n'entendant exactement les mêmes sons que son voisin.

Ce dispositif, Gance n'eut guère l'occasion de le mettre en œuvre, les producteurs de Cyrano et d'Artagnan lui en refusant les moyens.

Relisons Dumas. Sommes-nous encore dans une forme de pré-cinéma ou bien déjà dans un monde virtuel matérialisé ? « La lorgnette rapprochait tellement les objets à ses yeux, que deux ou trois fois Hoffmann étendit la main, croyant saisir Arsène qui ne paraissait plus être au bout du verre qui la reflétait, mais bien entre les deux verres de la lorgnette. " (La femme au collier de velours) Ce saisissement est en effet saisissant. Sans doute la réalisation de ce fantasme est-elle justifiée par la folie qui presse le cerveau de notre héros. Mais elle exprime encore et surtout une tension propre à l'écriture dumasienne. Écrire en sorte que l'imaginaire devienne la réalité même de ce que l'on est en train de vivre. Pour, non pas adapter, mais interagir avec Dumas, il faut qu'un cinéaste désire aller plus loin que le cinéma, au-delà des possibles que le cinéma avait déjà actualisés.

En 1963, alors qu'il s'efforce de sauver Cyrano et d'Artagnan de l'incompréhension de ses producteurs, Gance participe à un débat sur l'avenir du cinéma (dans le cadre du club Saint Gobain). Il imagine un état du cinéma où les actions seraient projetées dans un espace en trois dimensions, sans support et sans écran. Les personnages se déplaceraient dans le vide, sans autre appui que l'air. S'agit-il d'un espace stéréoscopique dit en 3 D ? Non, l'espace en trois dimensions du cinéma ne supprime pas l'écran. La stéréoscopie produit seulement l'illusion que l'action se déroule aussi en profondeur (et la simple image plane en $2 \mathrm{D}$ n'a pas attendu la $3 \mathrm{D}$ pour produire une telle illusion). Alors, de quel type d'espace s'agit-il? Je dirais qu'il s'agit de notre espace vécu au quotidien mais revisité et habité par l'imaginaire. Cet espace ne comporte pas d'images matérielles ou matérialisées par quelque procédé technique que ce soit. C'est le cerveau du spectacteur qui projette des images dans l'espace Et si nous prolongeons la vision de Gance, nous aboutissons à ceci : l'espace imaginé par Gance est un espace sans autre limite - si c'en est une - que l'horizon, un espace où le spectaCteur pourrait circuler et agir au même titre que les autres protagonistes de la fiction. L'imaginaire se matérialiserait dans un espace qui s'apparenterait au nôtre. C'est le rapport au temps - la quatrième dimension - qui ferait la différence. On pourrait convoquer simultanément plusieurs temporalités, se déplacer dans le temps comme dans l'espace. Le temps serait converti en espace.

Cette utopie aurait-elle trouvé un lieu - et une réponse technique - dans les systèmes de réalité virtuelle où l'interacteur se déplace dans des mondes simulés équipé de casques et de lunettes stéréoscopiques, de combinaisons et de gants de données? On pourrait être tenté d'interpréter la vision de Gance comme une anticipation des dispositifs de réalité virtuelle. Un fait troublant nous en empêche cependant: si Gance a multiplié le dépôt de 
brevets au cours de sa carrière, aucun dispositif technique ne se profile à l'horizon de sa vision. C'est que l'imaginaire ne relève pas de l'illusion. On peut penser a contrario que les mondes issus de la réalité virtuelle esquivent la question de l'imaginaire en le résorbant dans une réalité simulée qui ne vaut que par son coefficient de "réalisme ", c'est-à-dire par son degré de conformité à des représentations préexistantes.

L'imaginaire ne relève pas de l'illusion au sens où il n'a nul besoin de la restitution de sensations simulées pour exister, restitution qui suppose des moyens matériels d'actualisation, c'est-à-dire des dispositifs techniques. Ce n'est pas que l'imaginaire ignore les sensations, c'est qu'il les redéploie à un autre niveau, celui des synesthésies et des associations qui renvoient à une expérience réactivée du vécu. Il s'agit plutôt de partir des sensations du spectaCteur dans le désir d'agir qu'on a stimulé en lui et de provoquer un imaginaire de l'action. L'expérience spectatorielle devient alors une expérience de dédoublement. On est soi et un autre à la fois. L'activation de l'imaginaire produit des images immatérielles qui n'existent que dans le cerveau de chaque spectateur. Il ne s'agit pas de donner plus de réalité à l'illusion réaliste, comme entendent le faire les dispositifs de réalité virtuelle, mais à l'imaginaire.

L'écran constitue bien un obstacle qu'il faut abolir pour que l'imaginaire devienne enfin réalité. Mais ce processus doit rester à l'état de tension et d'intention pour que l'imaginaire demeure actif. Travailler le décalage entre image matérielle et image immatérielle, c'est renforcer la fabrique d'images dans le cerveau du spectacteur. L'imaginaire n'est pas condamné à la représentation. La vision de Gance anticiperait alors un nouvel état de l'imaginaire où la représentation matérialisée sur l'écran jouerait un rôle d'intensification et d'accélération de l'activité imageante du spectaCteur.

Il ne s'agit pas pour nous de tenir l'épée de Cyrano ou de d'Artagnan. Il s'agit de se projeter dans leur action comme si nous agissions à leur place. C'est dans ce " comme si » que les puissances de l'art sont susceptibles de se déployer, en littérature comme en cinéma.

\section{RÉSUMÉS}

Donner à lire pour imaginer, c'est montrer par l'écriture, c'est faire voir ce qui serait même interdit à la vue. L'écriture tend vers la transparence de la description. Les images générées par l'écriture sont à vivre. L'observateur se transforme très vite en acteur. La littérature donne à imaginer mais le cinéma, jusqu'à un certain point, concrétise cet imaginaire. L'imagination ne se laisse pas pour autant résorber par la perception qui se situe entre sens de la vue et sens haptique. La vue fait naître le désir de toucher : non le passage à l'acte mais le désir de l'acte. La limite de l'art se situe bien dans un au-delà du regard. D'où la place incontournable de l'imaginaire pour figurer le sens haptique, cet au-delà du regard et de l'action.

What imagine means

To give something to read to make you imagine, is to show through the writing, to make you see what would even be forbidden to the sight. Writing aims at the transparency of description. The images created by the writing are meant to be lived. The observer becomes soon an actor. 
Literature gives you to imagine but the cinema, up to a point, makes this imaginary world become real. The imagination however doesn't let itself be absorbed by the perception that is between the sense of sight and the haptic sense. To see makes you want to touch: you don't actually do it but you wish to. The limits of art indeed are situated somewhere beyond the eyes that see. Hence the predominant role of imagination to represent the haptic sense.

\section{AUTEUR}

\section{GÉRARD LEBLANC}

Professeur émérite à l'Ecole Nationale Supérieure Louis-Lumière et chercheur à l'I.R.C.A.V. Il a publié : Quand l'entreprise fait son cinéma (PUV-Cinéthique, 1983), Le monde en suspens (Hitzeroth, 1987), Le double scénario chez Fritz Lang (avec Brigitte Devismes, Armand Colin, 1991), Georges Franju, une esthétique de la déstabilisation (Créaphis, 1992), Scénarios du réel (2 volumes, L'Harmattan, 1997), L'EntreVues (avec Jean-Daniel Pollet, L'oeil, 1998), Cinéma et dernières technologies (avec Frank Beau et Philippe Dubois, De Boeck, 1998), Trajectoires (L'oeil, 2001), Presque une conception du monde (Créaphis, 2007), Les yeux au bout des doigts (Médias Création Recherche, 2010), Numérique et transesthétique (avec Sylvie Thouard, Presses Universitaires du Septentrion, 2012). 